\title{
Nickel: The time-reversal symmetry conserving partner of iron on a chalcogenide topological insulator
}

\author{
M. Vondráček, ${ }^{1, *}$ L. Cornils, ${ }^{2}$ J. Minár, ${ }^{3,4}$ J. Warmuth, ${ }^{2}$ M. Michiardi, ${ }^{5}$ C. Piamonteze, ${ }^{6}$ L. Barreto,,${ }^{5, \dagger}$ J. A. Miwa, ${ }^{5}$ \\ M. Bianchi, ${ }^{5}$ Ph. Hofmann, ${ }^{5}$ L. Zhou, ${ }^{2, \ddagger}$ A. Kamlapure, ${ }^{2}$ A. A. Khajetoorians, ${ }^{2,}{ }_{\S}^{\S}$ R. Wiesendanger, ${ }^{2}$ J.-L. Mi, ${ }^{7, \pi}$ \\ B.-B. Iversen, ${ }^{7}$ S. Mankovsky, ${ }^{3}$ St. Borek, ${ }^{3}$ H. Ebert, ${ }^{3}$ M. Schüler, ${ }^{8}$ T. Wehling, ${ }^{8}$ J. Wiebe, ${ }^{2}$ and J. Honolka ${ }^{1}$ \\ ${ }^{1}$ Institute of Physics of the Czech Academy of Sciences, Na Slovance 2, CZ-182 21 Prague, Czech Republic \\ ${ }^{2}$ Department of Physics, University of Hamburg, Jungiusstrasse 11, D-20355 Hamburg, Germany \\ ${ }^{3}$ Department of Chemistry, University of Munich, Butenandtstrasse 5-13, D-81377 München, Germany \\ ${ }^{4}$ New Technologies Research Center, University of West Bohemia, Univerzitní 8, CZ-306 14 Plzeñ, Czech Republic \\ ${ }^{5}$ Department of Physics and Astronomy, Interdisciplinary Nanoscience Center, Aarhus University, DK-8000 Aarhus C, Denmark \\ ${ }^{6}$ Swiss Light Source, Paul Scherrer Institut, CH-5232 Villigen PSI, Switzerland \\ ${ }^{7}$ Center for Materials Crystallography, Department of Chemistry and iNANO, Aarhus University, \\ Langelandsgade 140, DK-8000 Aarhus C, Denmark \\ ${ }_{8}^{8}$ Institute for Theoretical Physics, Bremen Center for Computational Material Science, University of Bremen, D-28359 Bremen, Germany
}

(Received 7 April 2016; revised manuscript received 13 July 2016; published 11 October 2016)

\begin{abstract}
We report on the quenching of single $\mathrm{Ni}$ adatom moments on Te-terminated $\mathrm{Bi}_{2} \mathrm{Te}_{2} \mathrm{Se}_{\text {and }} \mathrm{Bi}_{2} \mathrm{Te}_{3}$ topological insulator surfaces. The effect is noted as a missing $\mathrm{x}$-ray magnetic circular dichroism for resonant $L_{3,2}$ transitions into partially filled $\mathrm{Ni} 3 d$ states of theory-derived occupancy $n_{d}=9.2$. On the basis of a comparative study of $\mathrm{Ni}$ and Fe using scanning tunneling microscopy and $a b$ initio calculations, we are able to relate the element specific moment formation to a local Stoner criterion. Our theory shows that while Fe adatoms form large spin moments of $m_{s}=2.54 \mu_{B}$ with out-of-plane anisotropy due to a sufficiently large density of states at the Fermi energy, Ni remains well below an effective Stoner threshold for local moment formation. With the Fermi level remaining in the bulk band gap after adatom deposition, nonmagnetic Ni and preferentially out-of-plane oriented magnetic $\mathrm{Fe}$ with similar structural properties on $\mathrm{Bi}_{2} \mathrm{Te}_{2} \mathrm{Se}$ surfaces constitute a perfect platform to study the off-on effects of time-reversal symmetry breaking on topological surface states.
\end{abstract}

DOI: 10.1103/PhysRevB.94.161114

Three-dimensional topological insulators (TIs) are insulating in the bulk but host conductive topological surface states (TSSs), which follow a Dirac-like dispersion relation with spin and orbital momentum degrees of freedom chirally locked at a right angle [1,2]. As a consequence, currents for momenta $+\vec{k}$ and $-\vec{k}$ carry opposite spin, and $180^{\circ}$ backscattering is suppressed. While this particular topological spin texture is protected by time-reversal symmetry immanent in TIs, it is vulnerable to perturbations which are odd under time-reversal operation. The intimate tie-up between TI phase and time-reversal symmetry is presently the subject of intense experimental and theoretical efforts to understand the response of TSSs to time-symmetry violating magnetic exchange fields $\vec{H}_{\text {Ex }}$ introduced by magnetic guest atoms. For $\vec{H}_{\text {Ex }}$ oriented perpendicular to the surface plane in a stable manner, a gap at the Dirac point (DP) should appear as a signature of losing TSS properties [3,4].

\footnotetext{
*vondrac@fzu.cz

${ }^{\dagger}$ Present address: Centro de Ciências Naturais e Humanas, Universidade Federal do ABC, Santo André 09210-580, SP, Brazil.

${ }^{\ddagger}$ Present address: Max Planck Institute for Solid State Research, D-70569 Stuttgart, Germany.

${ }^{\S}$ Present address: Institute for Molecules and Materials (IMM), Radboud University, 6525 AJ Nijmegen, The Netherlands.

"Present address: Institute for Advanced Materials, School of Materials Science and Engineering, Jiangsu University, Zhenjiang 212013, China.
}

In the search for model systems, local spin and orbital moment formation, magnetocrystalline anisotropy of $3 d$ bulk and surface guest atoms, and charge doping were studied, particularly for prototypical bismuth chalcogenide TI host materials [5-8], where the nonvanished average exchange fields $\left\langle\vec{H}_{\text {Ex }}\right\rangle$ can be stabilized, e.g., by carrier-mediated exchange interactions $J_{3 \mathrm{D} / 2 \mathrm{D}}$ between guest atoms [9-11]. Dilute surface adatoms are promising since direct experimental access to structural and electronic properties using scanning tunneling microscopy (STM) and angle-resolved photoemission spectroscopy (ARPES) is retained. Here, ARPES reported intact TSSs [7], according to a simple picture of thermally fluctuating local moments $\left(J_{2 \mathrm{D}} \ll k_{B} T\right)$ giving $\left\langle\vec{H}_{\mathrm{Ex}}\right\rangle=0$ on the time scale required for a spin-flip process. In contrast, a recent low-temperature scanning tunneling spectroscopy (STS) study of local TSS scattering processes claims that paramagnetic but weakly coupled $3 d$ adatom moments are able to locally produce exchange fields on transient time scales sufficient to gap the TI phase [12]. A full understanding is complicated by the presence of the adatoms' nonmagnetic resonant scattering potential $U$, which, as in other Dirac materials such as graphene and $d$-wave superconductors, can counteract a moment-induced gap formation at the DP $[13,14]$.

In this Rapid Communication we report on an efficient quenching mechanism of single $\mathrm{Ni}$ adatom moments on $\mathrm{Te}$ terminated $\mathrm{Bi}_{2} \mathrm{Te}_{2} \mathrm{Se}$ and $\mathrm{Bi}_{2} \mathrm{Te}_{3}$ surfaces, despite a partially filled $\mathrm{Ni} 3 d$ shell with occupancy $n_{d}=9.2$. Our $a b$ initio calculations explain this surprising behavior in the framework of a local Stoner criterion, which predicts $\mathrm{Fe}$ adatoms to form large spin moments of $m_{s}=2.54 \mu_{B}$ with out-of-plane 
anisotropy, while $\mathrm{Ni}$ remains below an effective Stoner threshold for local moment formation. With the DP remaining in the $\mathrm{Bi}_{2} \mathrm{Te}_{2} \mathrm{Se}$ band gap for both elements $\mathrm{Ni}$ and $\mathrm{Fe}$, these systems are ideal to study magnetism-dependent TSSdominated transport, both macroscopically [15] and on the atomic scale via STS [12].

Our results are based on a thorough determination of the structural, electronic, and magnetic properties of $\mathrm{Ni}$ and $\mathrm{Fe}$ adatoms on $\mathrm{Bi}_{2} \mathrm{Te}_{2} \mathrm{Se}$ and $\mathrm{Bi}_{2} \mathrm{Te}_{3}$, combining local STM $[16,17]$ with $\mathrm{X}$-ray magnetic circular dichroism (XMCD) spectroscopy (X-Treme beamline, SLS [18]) and ARPES (SGM3 beamline at ASTRID2 synchrotron radiation facility [19]). Experiments were carried out with $\mathrm{Bi}_{2} \mathrm{Te}_{3}$ single crystals grown by the method described in Ref. [20] and with $\mathrm{Bi}_{2} \mathrm{Te}_{2} \mathrm{Se}$ single crystals identical to those used in Ref. [21]. For the latter crystals, four-point probe measurements have proven surface-dominated transport with a high bulk resistivity at low temperatures [15]. The crystals were cleaved in ultrahigh vacuum at room temperature using scotch tape, followed by cooling down to temperatures below $100 \mathrm{~K}$ within $25 \mathrm{~min}$ for ARPES, $20 \mathrm{~min}$ for XMCD, and within $5 \mathrm{~min}$ for STM measurements. Dilute amounts of $\mathrm{Ni}$ and $\mathrm{Fe}$ in the range of a percentage of a monolayer (ML, number of atoms per surface unit cell) were subsequently deposited from thoroughly outgassed and calibrated $e$-beam evaporators onto the cold substrate $(T=2 \mathrm{~K}$ for XMCD, $T=7 \mathrm{~K}$ for STM, and $T \approx 90 \mathrm{~K}$ for ARPES), in order to prevent clustering or subsurface diffusion of $\mathrm{Ni}$ and $\mathrm{Fe}$ atoms [22].

Figure 1(a) shows an STM topograph of the pristine $\mathrm{Bi}_{2} \mathrm{Te}_{3}$ surface after cleavage, which happens at the van der Waals bonds between the quintuple layers (QLs) (see the inset) leading to a Te-terminated surface. It shows the well-known defects in the Te layers of the topmost QL [23,24]. Similar studies on $\mathrm{Bi}_{2} \mathrm{Te}_{2} \mathrm{Se}$ crystals [see the Supplemental Material (SM) [25]] show $12 \% \pm 3 \%$ of $\mathrm{Se}_{\mathrm{Te}}$ antisite defects in the surface Te layer. Apart from those infrequent $\mathrm{Se}_{\mathrm{Te}}$ defects, the sequence of the topmost two layers of $\mathrm{Bi}_{2} \mathrm{Te}_{3}$ and $\mathrm{Bi}_{2} \mathrm{Te}_{2} \mathrm{Se}$ is thus identical and we expect similar adsorption geometries and magnetic properties for the $3 d$ atoms deposited on both Teterminated substrates. Figure 1(b) shows the STM topograph after deposition of $1 \% \mathrm{ML} \mathrm{Ni}$. In addition to the features from the intrinsic defects in the topmost Te layers (black circle), there is a statistical distribution of defects stemming from $\mathrm{Ni}$ adatoms which have an apparent height of $25-110 \mathrm{pm}$, depending on the bias voltage. Closer inspection reveals that the $\mathrm{Ni}$ atoms have two distinct appearances due to adsorption on either of the two hollow sites of the surface Te lattice [Fig. 1(c); see the lines]. Considering that the clover-shaped subsurface defects are attributed to $\mathrm{Bi}_{\mathrm{Te}}$ antisites residing in the fifth layer (Te) of the first QL [24,26] and their position relative to the surface Te atoms (see the lines), we can assign the shallower $\mathrm{Ni}$ atom to $\mathrm{Ni}_{\text {hcp }}$ and the higher one to $\mathrm{Ni}_{\text {fcc }}$. They have a strongly different relative abundance $\mathrm{Ni}_{\text {hcp }}: \mathrm{Ni}_{\text {fcc }}$ of 0.2. For $\mathrm{Fe}$ on $\mathrm{Bi}_{2} \mathrm{Te}_{3}$, our STM experiments confirm the previously revealed hollow site adsorption, appearance in STM, and relative abundance $\mathrm{Fe}_{\mathrm{hcp}}$ : $\mathrm{Fe}_{\mathrm{fcc}}$ of 0.7 [8]. Very similar results are found for the adsorption of the two elements on $\mathrm{Bi}_{2} \mathrm{Te}_{2} \mathrm{Se}$ (not shown).

In order to determine the vertical position of $\mathrm{Ni}$ for the electronic structure calculations shown below, we performed
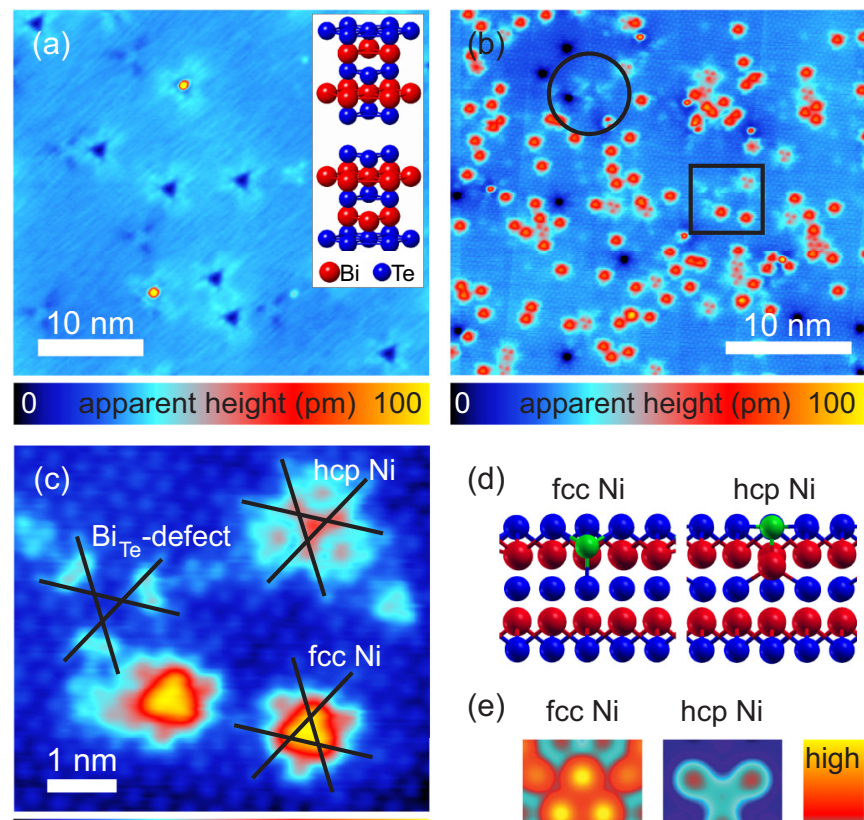

(e)

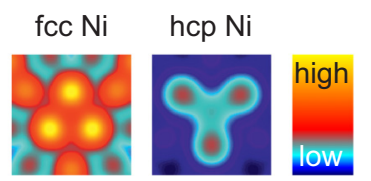

FIG. 1. (a) $\mathrm{STM}$ topograph of the pristine $\mathrm{Bi}_{2} \mathrm{Te}_{3}$ surface $(U=$ $350 \mathrm{mV}, I=20 \mathrm{pA})$. The inset illustrates two QLs of the crystal structure. (b) Overview image of $\approx 1 \% \mathrm{ML} \mathrm{Ni}$ (red dots and clover-shaped defects) on $\mathrm{Bi}_{2} \mathrm{Te}_{3}(U=-995 \mathrm{mV}, I=400 \mathrm{pA})$. The black circle highlights a typical $\mathrm{Bi}_{\mathrm{Te}}$ antisite defect. (c) Atomically resolved image of the area marked by the black square in (b) with single $\mathrm{Ni}$ atoms occupying two different adsorption sites and a $\mathrm{Bi}_{\mathrm{Te}}$ antisite defect $(U=-350 \mathrm{mV}, I=400 \mathrm{pA}$, composite of original and Fourier filtered topograph in order to enhance atomic contrast). The lines trace the surface Te atom rows. (d) Side views of the relaxed positions of $\mathrm{Ni}_{\text {fcc }}$ and $\mathrm{Ni}_{\text {hcp }}$ from DFT calculations (Bi atoms displayed red, Te blue). (e) Calculated STM topographs of $\mathrm{Ni}_{\mathrm{fcc}}$ and $\mathrm{Ni}_{\text {hcp }}(U=-400 \mathrm{mV})$. For both stackings, the $z$ scale covers the same range. All the STM measurements were performed at $T=4 \mathrm{~K}$.

density functional theory (DFT) calculations equivalent to those in Ref. [6]. We started from the two lateral positions of the adatoms revealed by STM [Fig. 1(c)] and then relaxed them [Fig. 1(d)]. While $\mathrm{Ni}_{\text {fcc }}$ strongly relaxes into the first Bi layer, $\mathrm{Ni}_{\text {hep }}$ sits in the surface Te layer and pushes the underlying Bi atom downward. Using these two adsorption geometries, we calculate STM images [Fig. 1(e)] with the Tersoff-Hamann approach [27] by evaluating the partial charge density in a range of $0.4 \mathrm{eV}$ below the Fermi energy $\left(E_{\mathrm{F}}\right)$ at $\sim 2.1 \AA$ above the surface. The images confirm the experimentally observed difference in height and appearance of $\mathrm{Ni}_{\text {fcc }}$ and $\mathrm{Ni}_{\text {hcp }}$ as well as the assignment to the adsorption sites. Our calculations for the case of Fe confirm previous results [8].

The top Te-Bi layer is identical for $\mathrm{Bi}_{2} \mathrm{Te}_{3}$ and $\mathrm{Bi}_{2} \mathrm{Te}_{2} \mathrm{Se}$ [see the structural models in Figs. 1(a) and 2(g) [28]], suggesting similar relaxed positions of $\mathrm{Ni}$ or $\mathrm{Fe}$. As a consequence, we expect the same chemical bonding and magnetic properties for those substrates, as confirmed by x-ray photoemission spectroscopy (XPS) and XMCD results (see below).

Surface-sensitive XPS of the Bi $5 d$ and Te $4 d$ core levels from pristine $\mathrm{Bi}_{2} \mathrm{Te}_{2} \mathrm{Se}$ in Figs. 2(a) and 2(d) develop strong shoulders upon deposition of 0.4 ML Fe [Figs. 2(b) and 2(e)] and 0.5 ML Ni [Figs. 2(c) and 2(f)], while the Se $3 d$ core 

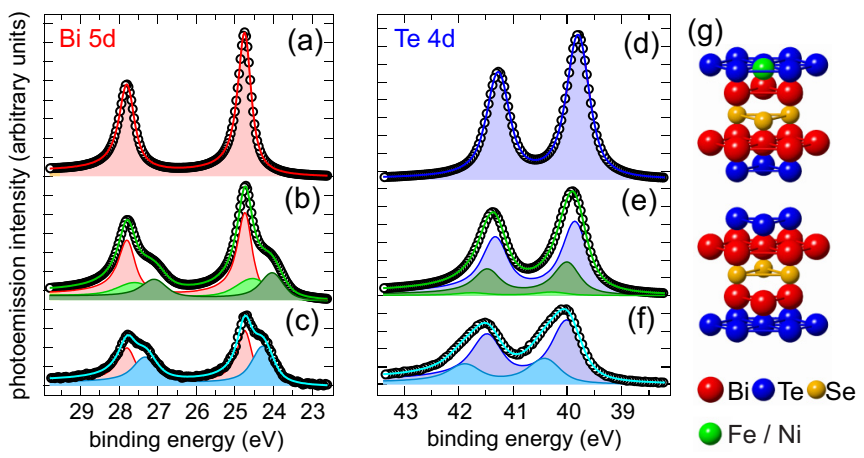

FIG. 2. Bi $5 d$ (left panel) and Te $4 d$ (right panel) XPS core level spectra at a photon energy $h v=100 \mathrm{eV}$ and $T \approx 90 \mathrm{~K}$ for pristine $\mathrm{Bi}_{2} \mathrm{Te}_{2} \mathrm{Se}[(\mathrm{a}),(\mathrm{d})]$, as well as after deposition of 0.4 ML Fe [(b), (e)] and 0.5 ML Ni [(c), (f)], respectively. Experimental data (circles) are shown together with fitted results (lines) decomposed into $\mathrm{Bi}$ and $\mathrm{Te}$ doublets (solid areas). (g) Illustration of the top two QLs of $\mathrm{Bi}_{2} \mathrm{Te}_{2} \mathrm{Se}$ with an adsorbed adatom.

level spectrum does not show significant changes in the line shape (see SM [25]). This is consistent with metal adatoms residing between the two topmost layers, where they cause strong chemical shifts in neighboring $\mathrm{Te}$ and $\mathrm{Bi}$ atoms, but not in Se [see Fig. 2(g)], because adatoms have little influence on the third subsurface layer. $\mathrm{Ni}$ and Fe similarly shift Bi $5 d$ (Te $4 d$ ) lines to lower (higher) binding energies, in accordance with a weakening of the polar $\mathrm{Bi}-\mathrm{Te}$ bond toward a more metallic bonding, as expected. The peak decomposition (see SM [25] for details) shown in Fig. 2 reveals that, while Ni deposition generates a single component shifted by $0.46 \mathrm{eV}$, $\mathrm{Fe}$ undoubtedly forms two distinct components shifted by 0.23 and $0.72 \mathrm{eV}$ with respect to the main pristine $\mathrm{Bi}$ doublet. Although coverages here are beyond the impurity regime, these two components are consistent with the STM results, which showed that Fe atoms sit directly above Bi atoms (hcp site, stronger chemical shift) and above empty spaces (fcc site, weaker chemical shift) with nearly equal probability. $\mathrm{Ni}$ atoms in contrast strongly prefer fcc sites leading to only one shifted component. The Te spectra in Fig. 2(b) show only one additional component in both cases $\mathrm{Ni}$ and $\mathrm{Fe}$, very likely because both fcc and hcp adatoms are embedded in a Te triangular geometry.

In order to study the doping effect of adatoms on the TSS, we performed ARPES for different coverages. Generally, the surface impurities lead to a downward energy shift of the TSS and bulk bands [28], which saturates at coverages in the percentage range of a ML. This indicates electron transfer from the adatom to the substrate. As shown in Fig. 3, at $\leqslant 1 \%$ coverages, only the linearly dispersing TSS crosses $E_{\mathrm{F}}$ located in the bulk band gap of $\mathrm{Bi}_{2} \mathrm{Te}_{3}$ and $\mathrm{Bi}_{2} \mathrm{Te}_{2} \mathrm{Se}$. Thus, these systems represent the very particular case where transport will be determined exclusively by the interaction of the TSS with the localized $3 d$ guest atom's charge and magnetic moment, which will be investigated in the following.

The late $3 d$ transition element $\mathrm{Ni}$ is known to be a borderline element for transitions between high-spin $3 d^{8} 4 s^{2}$ and lowspin $3 d^{9} 4 s^{1}$ configurations already in the free atom case or on weakly interacting graphitic substrates [29,30], and thus adatom electronic and magnetic ground state configurations
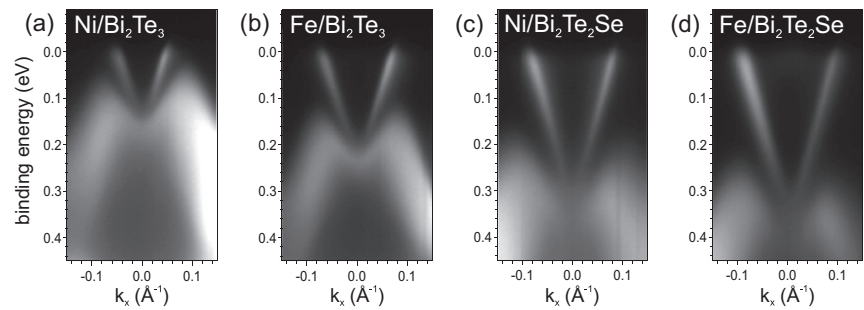

FIG. 3. ARPES data for $\approx 0.3 \% \mathrm{ML} \mathrm{Ni}$ on (a) $\mathrm{Bi}_{2} \mathrm{Te}_{3}$ and (c) $\mathrm{Bi}_{2} \mathrm{Te}_{2} \mathrm{Se}$, respectively, and $\approx 0.2 \% \mathrm{ML} \mathrm{Fe}$ on (b) $\mathrm{Bi}_{2} \mathrm{Te}_{3}$ and (d) $\mathrm{Bi}_{2} \mathrm{Te}_{2} \mathrm{Se}$, respectively. Spectra were taken at a photon energy $h v=$ $21 \mathrm{eV}$ and $T \approx 90 \mathrm{~K}$.

are highly sensitive to interactions with the host surface. For an analysis of the magnetic ground state properties on $\mathrm{Bi}_{2} \mathrm{Te}_{3}$ and $\mathrm{Bi}_{2} \mathrm{Te}_{2} \mathrm{Se}$, we performed $\mathrm{x}$-ray absorption spectroscopy (XAS) at the $L_{3,2}$ edges (Fig. 4), which provide information on the local electronic state, since the spectral shapes and resonant absorption intensities depend on the distribution of holes in the $3 d$ shell. For magnetic contrast we applied large magnetic fields of $B=6.8 \mathrm{~T}$ collinear to a positive ( + ) and negative $(-)$ circularly polarized x-ray beam, as depicted in Fig. 4(a). Differences in the respective absorption spectra $\sigma^{+}$and $\sigma^{-}$reflect the magnetic ground state of single $3 d$ atoms as described in an earlier work [6]. Figures 4(a) and 4(b) summarize Ni and Fe spectroscopy in the single impurity limit and corresponding DFT results based on the spin-polarized relativistic multiplescattering or Korringa-Kohn-Rostoker formalism using the coherent potential approximation [31], together with Fermi's golden rule [32]. All XAS data in Fig. 4 were measured at normal incidence $\theta=0^{\circ}$ in the total electron yield mode, and spectra are shown after $\mathrm{Bi}_{2} \mathrm{Te}_{2} \mathrm{Se}$ background subtraction. Before and after XAS characterization, the samples were carefully checked for oxygen contamination using the XAS at the $\mathrm{O} K$ edge.

Prominent resonant multiplet structures visible especially at the leading $L_{3}$ edge of $\mathrm{Ni}$ and $\mathrm{Fe}$ spectra are typical for partly filled localized $d$ shells weakly hybridized with their environments. The well-resolved multiplet structures in XAS serve as a fingerprint of the local $d$-shell configurations and show an exceptional agreement with the ones derived from DFT [right panels of Figs. 4(a) and 4(b)]. They correspond to unfilled $\mathrm{Ni}$ and $\mathrm{Fe} d$-shell occupancies $n_{d}=9.2$ and $n_{d}=6.9$, respectively. Strikingly, while Fe develops a local moment as reflected by the Fe XMCD signal $\left(\sigma^{+}-\sigma^{-}\right)$, Ni takes a nonmagnetic ground state [see the lower panels in Figs. 4(a) and 4(b)]. Corresponding spectroscopy data at a grazing incidence $\theta=60^{\circ}$ gave similar results for $\mathrm{Ni}$, while Fe spectra showed a pronounced out-of-plane magnetic easy axis with a magnetic anisotropy of $7 \pm 3 \mathrm{meV} /$ atom (see SM [25]), in agreement with Eelbo et al. [8]. Consistently, our DFT results in local moments of $m_{s}=0 \mu_{B}$ and $m_{l}=0 \mu_{B}$ for $\mathrm{Ni}$ and $m_{s}=2.54 \mu_{B}$ and $m_{l}=0.41 \mu_{B}$ for Fe. The moments are determined mainly by the $3 d$ shell since $4 s$ and $4 p$ contributions are negligible for $\mathrm{Ni}\left(m_{4 s}=0 \mu_{B}\right.$ and $m_{4 p}=$ $\left.0 \mu_{B}\right)$ and for $\mathrm{Fe}\left(m_{4 s}=0.026 \mu_{B}\right.$ and $\left.m_{4 p}=0.031 \mu_{B}\right)$. This quenching of the $\mathrm{Ni}$ atomic moment is a consequence of the interaction of the $3 d$ element with the top Te-Bi layer as the experimental finding is the same for binary $\mathrm{Bi}_{2} \mathrm{Te}_{3}$ (see $\mathrm{SM}$ [25]). 
(a) experiment
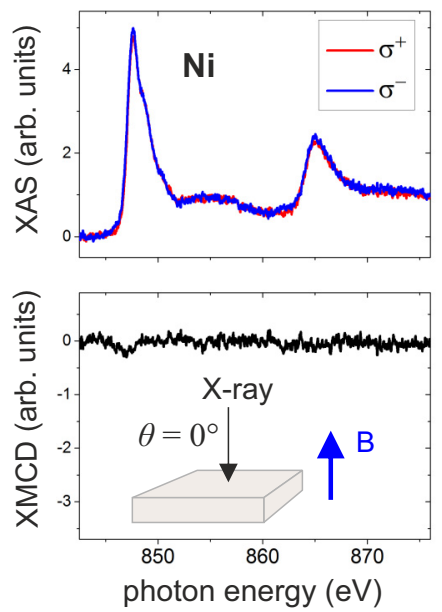

DFT theory


(b) experiment
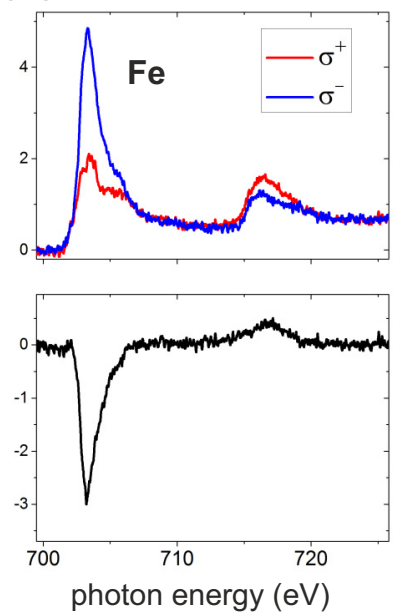

DFT theory
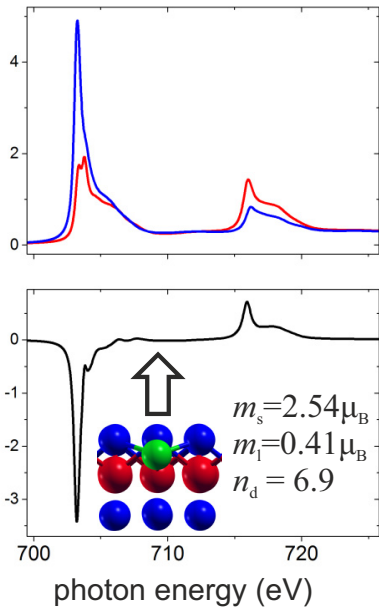

FIG. 4. (a) and (b) Ni and $\mathrm{Fe} L_{2,3}$ XAS data on $\mathrm{Bi}_{2} \mathrm{Te}_{2} \mathrm{Se}$ for positive $\left(\sigma^{+}\right)$and negative $\left(\sigma^{-}\right)$circular polarization in polar geometry $\left(\theta=0^{\circ}\right)$ and the resulting XMCD signals below (left panels). Measurements were done in the impurity regime ( $\approx 1 \%$ coverage) at low temperatures of $T=2 \mathrm{~K}$ and polar fields of $B=6.8 \mathrm{~T}$. Right panels show respective spectra calculated for $\mathrm{Bi}_{2} \mathrm{Te}_{3}$ on the basis of DFT theory using the relaxed fcc geometry shown as insets. Ni and Fe both show partially filled $d$ shells but only Fe develops finite local orbital and spin moments.

To gain an understanding of the mechanism responsible for the local Ni moment quenching in a partially filled $3 d$ shell, we compare the stability of the magnetic states of relaxed $\mathrm{Ni}$ and Fe atoms with our DFT calculations. The local densities of states (DOS) in Fig. 5 show that despite the localized character of the $\mathrm{Ni}$ and Fe $d$ electrons, the width of the partially occupied energy bands is rather large due to hybridization with the electronic states of the surrounding host atoms and is comparable to the width of the energy bands in $3 d$ itinerant ferromagnets. This result suggests the use of the Stoner criterion $\left(I \chi_{0}\right)^{k} \geqslant 1$ for the spontaneous formation of a spin moment on atom $k$ (Ni or Fe in our case), where $\left(I \chi_{0}\right)^{k}$ is the so-called Stoner product to analyze the atom of type $k$. For the most simple situations, $\left(I \chi_{0}\right)^{k}$ can be split into the Stoner integral $I^{k}$ and the enhanced spin susceptibility $\chi_{0}^{k}=2 \mu_{B} n^{k}\left(E_{\mathrm{F}}\right)$, where $n^{k}\left(E_{\mathrm{F}}\right)$ is the DOS at $E_{\mathrm{F}}$. For the more general case of an alloy and adatoms considered here, we express $\left(I \chi_{0}\right)^{k}$ using linear response theory, as suggested by Deng et al. [33] (see SM [25]).

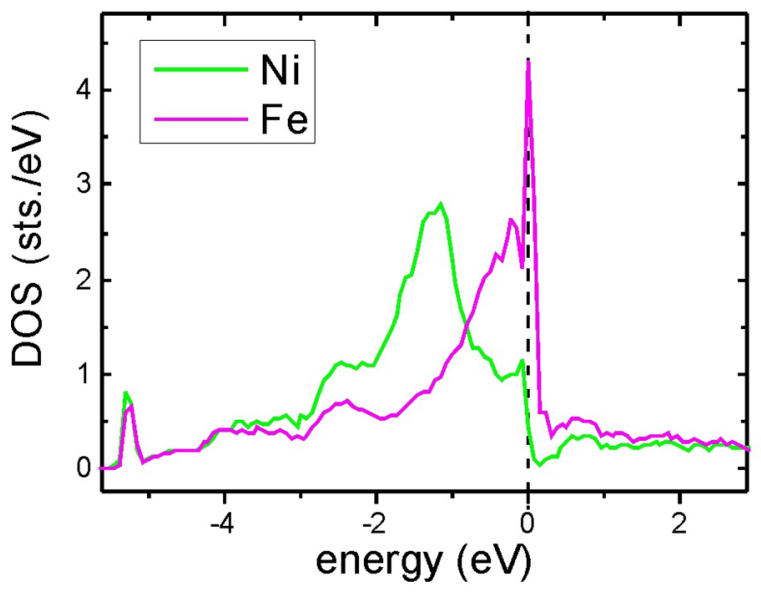

FIG. 5. Element-resolved total densities of states of $\mathrm{Ni}$ and $\mathrm{Fe}$ adatoms on $\mathrm{Bi}_{2} \mathrm{Te}_{3}$ calculated for the nonmagnetic state.
For Fe atoms, our self-consistent calculations give a Stoner product $\left(I \chi_{0}\right)^{\mathrm{Fe}}=2.68$ well above 1 , while for $\mathrm{Ni}$ we find only $\left(I \chi_{0}\right)^{\mathrm{Ni}}=0.78$. We thus can understand the quenching mechanism on the atomic level surprisingly well within the Stoner model, triggered by a critically low DOS at $E_{\mathrm{F}}$ on the $\mathrm{Ni}$ site close to the edge of the Ni $d$ band (Fig. 5).

In summary, we report on the complete quenching of $\mathrm{Ni}$ adatom moments situated on Te-terminated bismuth chalcogenide topological insulator surfaces. Comparing the adatom site and electronic properties of $\mathrm{Ni}$ with magnetic $\mathrm{Fe}$, our study provides a clear understanding of the quenching in the framework of a local Stoner theory. We find Fermi levels in the gap of $\mathrm{Bi}_{2} \mathrm{Te}_{2} \mathrm{Se}$ in the presence of adatoms, which are ideal for investigating topological insulators under nonmagnetic $\mathrm{Ni}$ and out-of-plane Fe moment perturbations. Under such well-defined magnetic off/on conditions, the recently claimed gap opening solely due to nonmagnetic resonant scattering potentials [34] can be verified using surface concepts.

The authors acknowledge financial support from the Czech Science Foundation (Grant No. GA14-30062S), Ministry of Education, Youth and Sports of the Czech Republic (Grants No. LM2011029 and No. LO1409), Deutsche Forschungsgemeinschaft (DFG) through SPP 1666 (under projects Eb154/26, WI 3097/2-1 and HO5150/1-2), Aarhus University Research Foundation and the VILLUM Foundation, COST Action MP1306 (EuSpec), and CENTEM PLUS (LO1402). J.Wa. and A.A.K. acknowledge support from the DFG via the Emmy Noether Program (KH324/1-1) and J.H. acknowledges support the Purkyně Fellowship program of the Czech Academy of Sciences. The X-ray absorption measurements were performed on the EPFL/PSI X-Treme beamline at the Swiss Light Source, Paul Scherrer Institut, Villigen, Switzerland. The research leading to these results has received funding from the European Community's Seventh Framework Programme (FP7/2007-2013) under Grant Agreement No. 312284. 
[1] M. Z. Hasan and C. L. Kane, Rev. Mod. Phys. 82, 3045 (2010).

[2] X.-L. Qi and S.-C. Zhang, Rev. Mod. Phys. 83, 1057 (2011).

[3] Q. Liu, C.-X. Liu, C. Xu, X.-L. Qi, and S.-C. Zhang, Phys. Rev. Lett. 102, 156603 (2009).

[4] J. Henk, M. Flieger, I. V. Maznichenko, I. Mertig, A. Ernst, S. V. Eremeev, and E. V. Chulkov, Phys. Rev. Lett. 109, 076801 (2012).

[5] L. A. Wray, S.-Y. Xu, Y. Xia, D. Hsieh, A. V. Fedorov, Y. S. Hor, R. J. Cava, A. Bansil, H. Lin, and M. Z. Hasan, Nat. Phys. 7, 32 (2011).

[6] J. Honolka, A. A. Khajetoorians, V. Sessi, T. O. Wehling, S. Stepanow, J.-L. Mi, B. B. Iversen, T. Schlenk, J. Wiebe, N. B. Brookes et al., Phys. Rev. Lett. 108, 256811 (2012).

[7] M. R. Scholz, J. Sánchez-Barriga, D. Marchenko, A. Varykhalov, A. Volykhov, L. V. Yashina, and O. Rader, Phys. Rev. Lett. 108, 256810 (2012).

[8] T. Eelbo, M. Waśniowska, M. Sikora, M. Dobrzański, A. Kozłowski, A. Pulkin, G. Autès, I. Miotkowski, O. V. Yazyev, and R. Wiesendanger, Phys. Rev. B 89, 104424 (2014).

[9] R. R. Biswas and A. V. Balatsky, Phys. Rev. B 81, 233405 (2010).

[10] D. A. Abanin and D. A. Pesin, Phys. Rev. Lett. 106, 136802 (2011).

[11] D. K. Efimkin and V. Galitski, Phys. Rev. B 89, 115431 (2014).

[12] P. Sessi, F. Reis, B. Thomas, K. A. Kokh, O. E. Tereshchenko, and M. Bode, Nat. Commun. 5, 5349 (2014).

[13] A. M. Black-Schaffer, A. V. Balatsky, and J. Fransson, Phys. Rev. B 91, 201411 (2015).

[14] P. Sessi, R. R. Biswas, T. Bathon, O. Storz, S. Wilfert, A. Barla, K. A. Kokh, O. E. Tereshchenko, K. Fauth, M. Bode et al., Nat. Commun. 7, 12027 (2016).

[15] L. Barreto, L. Kühnemund, F. Edler, C. Tegenkamp, J. Mi, M. Bremholm, B. B. Iversen, C. Frydendahl, M. Bianchi, and P. Hofmann, Nano Lett. 14, 3755 (2014).

[16] M. Bianchi, R. C. Hatch, Z. Li, P. Hofmann, F. Song, J. Mi, B. B. Iversen, Z. M. Abd El-Fattah, P. Löptien, L. Zhou et al., ACS Nano 6, 7009 (2012).

[17] P. Löptien, L. Zhou, A. A. Khajetoorians, J. Wiebe, and R. Wiesendanger, J. Phys.: Condens. Matter 26, 425703 (2014).

[18] C. Piamonteze, U. Flechsig, S. Rusponi, J. Dreiser, J. Heidler, M. Schmidt, R. Wetter, M. Calvi, T. Schmidt, H. Pruchova et al., J. Synchrotron Radiat. 19, 661 (2012).
[19] S. Hoffmann, C. Søndergaard, C. Schultz, Z. Li, and P. Hofmann, Nucl. Instrum. Methods Phys. Res., Sect. A 523, 441 (2004).

[20] M. Michiardi, I. Aguilera, M. Bianchi, V. E. de Carvalho, L. O Ladeira, N. G. Teixeira, E. A. Soares, C. Friedrich, S. Blügel, and P. Hofmann, Phys. Rev. B 90, 075105 (2014).

[21] J.-L. Mi, M. Bremholm, M. Bianchi, K. Borup, S. Johnsen, M. Søndergaard, D. Guan, R. C. Hatch, P. Hofmann, and B. B. Iversen, Adv. Mater. 25, 889 (2013).

[22] T. Schlenk, M. Bianchi, M. Koleini, A. Eich, O. Pietzsch, T. O. Wehling, T. Frauenheim, A. Balatsky, J.-L. Mi, B. B. Iversen et al., Phys. Rev. Lett. 110, 126804 (2013).

[23] P. Sessi, M. M. Otrokov, T. Bathon, M. G. Vergniory, S. S. Tsirkin, K. A. Kokh, O. E. Tereshchenko, E. V. Chulkov, and M. Bode, Phys. Rev. B 88, 161407 (2013).

[24] T. Bathon, S. Achilli, P. Sessi, V. A. Golyashov, K. A. Kokh, O. E. Tereshchenko, and M. Bode, Adv. Mater. 28, 2183 (2016).

[25] See Supplemental Material at http://link.aps.org/supplemental/ 10.1103/PhysRevB.94.161114 for STM of pristine $\mathrm{Bi}_{2} \mathrm{Te}_{2} \mathrm{Se}$, core level XPS data and fitting parameters, additional XAS and XMCD spectra, and details of DFT calculations.

[26] G. Wang, X.-G. Zhu, Y.-Y. Sun, Y.-Y. Li, T. Zhang, J. Wen, X. Chen, K. He, L.-L. Wang, X.-C. Ma et al., Adv. Mater. 23, 2929 (2011).

[27] J. Tersoff and D. R. Hamann, Phys. Rev. B 31, 805 (1985).

[28] S. Jia, H. Beidenkopf, I. Drozdov, M. K. Fuccillo, J. Seo, J. Xiong, N. P. Ong, A. Yazdani, and R. J. Cava, Phys. Rev. B 86, 165119 (2012).

[29] K. Godehusen, T. Richter, P. Zimmermann, and M. Martins, Phys. Rev. Lett. 88, 217601 (2002).

[30] V. Sessi, S. Stepanow, A. N. Rudenko, S. Krotzky, K. Kern, F. Hiebel, P. Mallet, J.-Y. Veuillen, O. Šipr, J. Honolka et al., New J. Phys. 16, 062001 (2014).

[31] H. Ebert, D. Ködderitzsch, and J. Minár, Rep. Prog. Phys. 74, 096501 (2011).

[32] J. Minár and H. Ebert, Appl. Phys. A 78, 847 (2004).

[33] M. Deng, H. Freyer, J. Voitländer, and H. Ebert, J. Phys.: Condens. Matter 13, 8551 (2001).

[34] J. Sánchez-Barriga, A. Varykhalov, G. Springholz, H. Steiner, R. Kirchschlager, G. Bauer, O. Caha, E. Schierle, E. Weschke, A. A. Ünal et al., Nat. Commun. 7, 10559 (2016). 